\title{
Optical Fibre-based Environmental Sensors Utilizing Wireless Smart Grid Platform
}

\author{
Minglong Zhang ${ }^{1}$, Kin Kee Chow ${ }^{2 *}$, and Peter Han Joo Chong ${ }^{1}$ \\ ${ }^{1}$ Department of Electrical and Electronic Engineering, Auckland University of Technology, \\ New Zealand \\ ${ }^{2}$ School of Engineering, Manchester Metropolitan University, United Kingdom \\ *K.Chow@mmu.ac.uk
}

\begin{abstract}
With the advent and development of smart grid in recent years, traditional power grid is undergoing a profound revolution. By utilizing modern wireless technology and sensor, wireless smart grid (WSG) can effectively solve many hard and haunting issues in traditional grid, such as high maintenance costs, poor scalability, low efficiency and stability. In a WSG, data are collected by sensors at first and then transmitted to base station through wireless network. After receiving those data, corresponding actions are executed by control center. We present the integration of optical fibre-based sensor to WSG platform for real-time environmental monitoring. As a proof-of-concept, an optical fibre sensor for refractive index (RI) sensing of fresh water is adopted. The sensing mechanism relies on the reflectance at the fibre interface, where the intensity of the reflected spectra is registered corresponding to the change of the RI of the ambient environment. A sensitivity of $29.3 \mathrm{~dB} / \mathrm{RIU}$ is achieved for the fabricated fibre sensor within the RI range of 1.33-1.46, and the acquired data is transmitted through wireless smart meters.
\end{abstract}

Keywords: Smart grid; wireless sensor networks; optical fibre sensors; environmental monitoring.

\section{Introduction}

Current power grid system becomes more and more complicated, as well as out of date in many aspects all over the world. Being lack of modern technology, large conventional power grids suffer from energy loss, overload condition, power quality issues, poor peak load management and time wastage on manual operation. To deal with those problems, many works have been done to upgrade our electrical grid to smart grid. By relying on latest technologies, the goal of smart grid is to provide more reliable distribution, improve fault detection and allow self-healing of the network without the intervention of technicians, create greater efficiencies in monitoring and load adjustments based on peak using times and locations, provide better security to the grid, and to empower the consumer to be able to better manage their usage and costs [1].

Compared with traditional electricity system, smart grids share several characteristics, mainly involving increased use of information and automatic control technology, integration of advanced appliances and consumer devices, dynamic 
optimization of grid operations and resources, deployment of smart technologies for metering, monitoring and communications concerning grid operations and status. Therefore, a smart grid mainly consists of smart meters, sensors, monitoring system, communication system and data management system [1].

Wireless communication and networking, with its low cost, high flexibility and less complexity, is widely adopted in many smart grids. In a wireless smart grid, data is firstly collected by different types of sensors and then sent to base station through wireless networks. A smart grid sensor, which serves as a detection node, can enable the remote monitoring of equipment such as transformers and power lines. For instance, smart grid sensors can monitor weather conditions and power line temperature, which can then be used to calculate the line's carrying capacity. This process is called dynamic line rating and it enables power companies to improve the efficiency of power transmission lines $[2,3]$.

In this paper, we propose and demonstrate the integration of optical fibre based sensors with the wireless smart grid platform for real-time remote environmental monitoring. As a proof-of-concept, optical fibre-based refractive index (RI) sensor for fresh water is investigated. Fig. 1 shows the overview of wireless smart grid architecture. The sensing probe was fabricated by first fusion splicing a multimode fibre (MMF) segment to a single mode fibre (SMF). The other end of the MMF segment is then cleaved to a certain length to form a joint fibre segment. Carbon nanotubes (CNTs) were then deposited onto the cleaved end face of the MMF segment using an optical deposition procedure [4, 5]. The behavior of the sensing probe to RI perturbations in the external environment was then characterized by a variation in intensity of the spectral features in the output spectrum with little fluctuations in their wavelengths. The deposited CNTs which are known to be a material with unique optical properties, such as a high RI, enabled the sensing probe to continuously measure a wide range of RIs [6-7]. The sensing probe also displayed little variation in intensity when it was immersed in a solution of fixed RI over a period of time and can be concluded to exhibit good stability. 


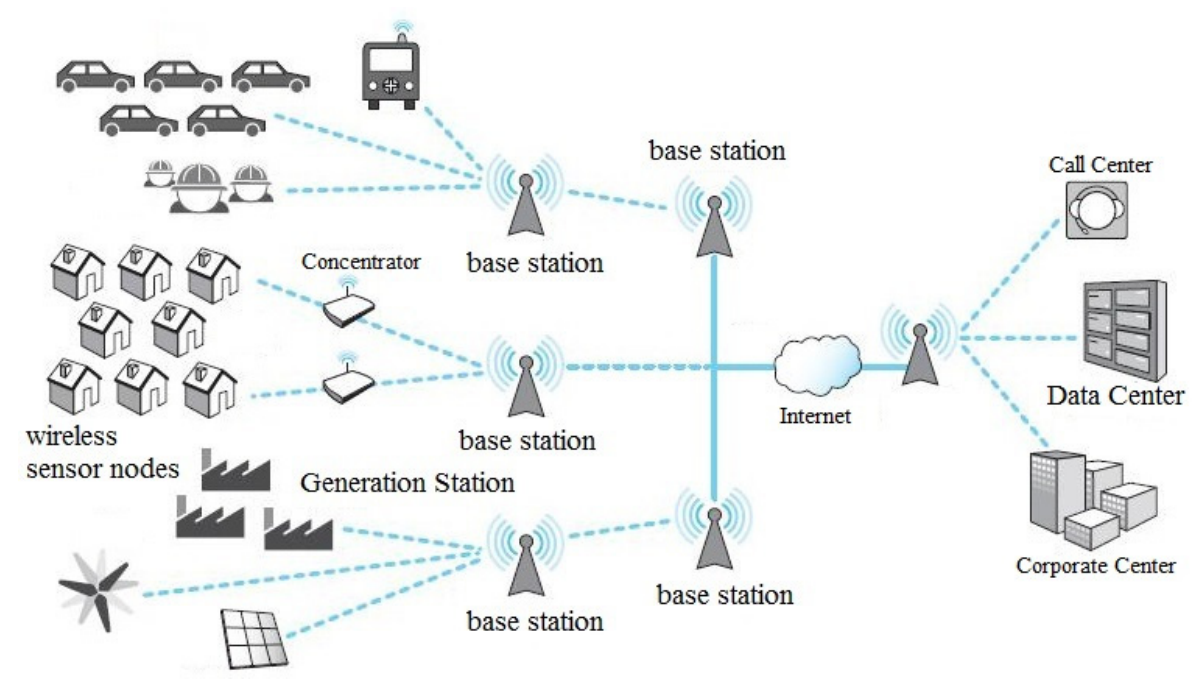

Fig. 1. Overview of wireless smart grid architecture.

\section{Fabrication of Sensing Probe}

In our experiment, a MMF fibre with core and cladding diameters of $105 \mu \mathrm{m}$ and 125 $\mu \mathrm{m}$, respectively, is first fusion spliced to a standard SMF. The MMF is then cleaved to a length of $21 \mathrm{~mm}$ from the SMF-MMF splice point to form the SMF-MMF joint fibre segment. To deposit CNTs onto the cleaved MMF end face of the joint fibre segment, a CNT solution is prepared by first dispersing CNT powder into a fixed volume of dimethylformamide (DMF) solution and then sonicating the resulting solution in an ultrasonic water bath for approximately 30 hours. Optical deposition of CNTs onto the end face of the MMF is carried out and the structure of the fibre sensor head is illustrated in Fig. 2(a) [4, 5]. Fig. 2(b) shows the microscopy image of the MMF end of the joint fibre segment after the deposition of CNTs. As can be seen, CNTs are deposited over the cleaved end face of the MMF. The output spectrum of the joint fibre segment before (dashed line) and after (solid line) the optical deposition process is shown in Fig. 2(c). The increase in intensity of the reflected spectrum is to be expected as the CNTs would vary the amount of reflectance, hence the change of the ambient RI can be registered. 


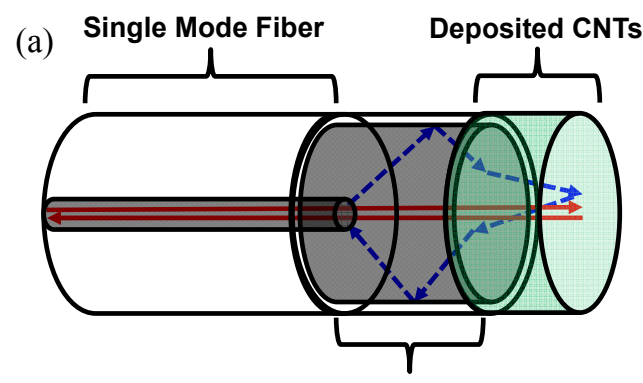

(b)
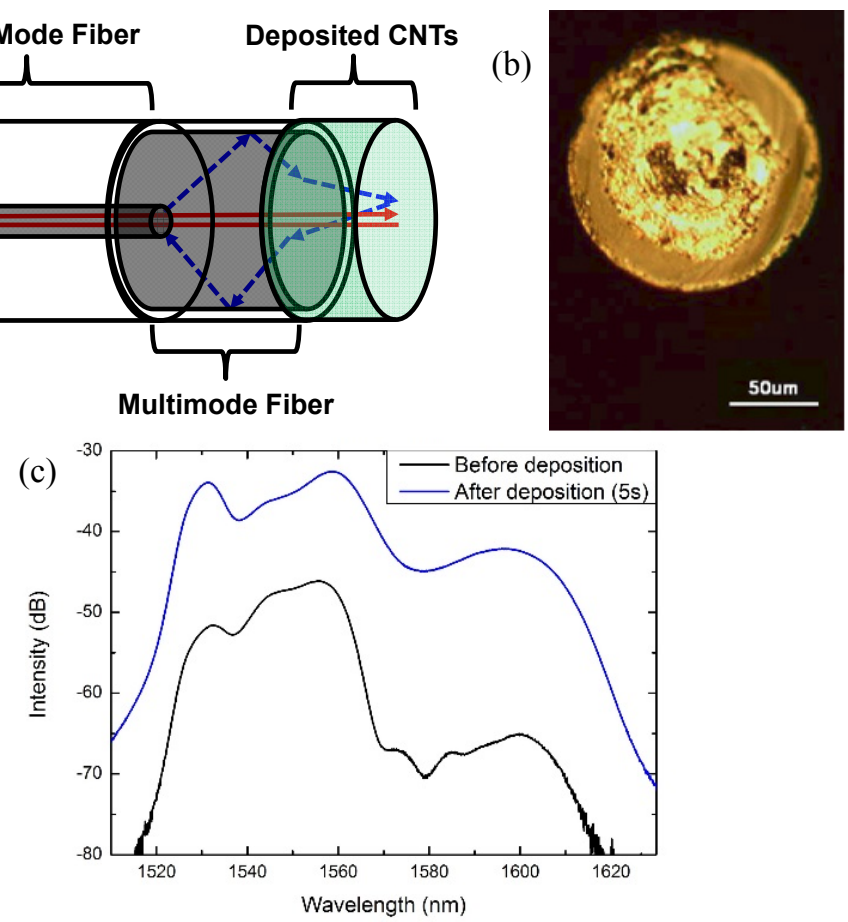

Fig. 2. (a) Schematic illustration of the sensing probe fabricated through depositing carbon nanotubes (CNTs) onto the cleaved multimode fiber end face of the joint fiber segment; (b) microscopy of CNTs deposition onto the MMF end of the joint fiber segment; and (c) the reflected spectrum of the joint fiber segment before and after

CNTs deposition.

\section{Experiment and Discussion}

In order to carry out the RI characterization, test solutions of different sugar concentrations and hence different RIs, are used to simulate perturbations in RI of the external environment of the sensing probe. These test solutions are prepared by first dissolving sugar into de-ionized (DI) water until a saturated sucrose solution is obtained. Fixed volumes of the saturated sucrose solution are then drawn out and diluted with different volumes of DI water to obtain the test solutions with different RIs. A commercially available refractometer is used to measure the RI of each test solution. To minimize the cross-coupling effects between RI and temperature, the characterization process is carried out in a thermally stable environment at an ambient temperature of $23.7 \pm 0.1{ }^{\circ} \mathrm{C}$. The SMF end of the sensing probe is connected to a broadband source via a circulator as shown in Fig. 3. The back reflected light from the sensing probe is monitored by an optical spectrum analyser (OSA). $1 \mathrm{ml}$ of each of the test solutions is dropped on a glass slide and the sensing probe is then completely immersed in the test solution and the corresponding variation in intensity of the spectral 
features in the output spectra was recorded on the OSA. The sensing probe is properly rinsed with DI water and allowed to dry in between test solutions. It is ensured that the reflected spectrum when the sensing probe was exposed to air was recovered before the sensing probe was immersed in the next test solution.

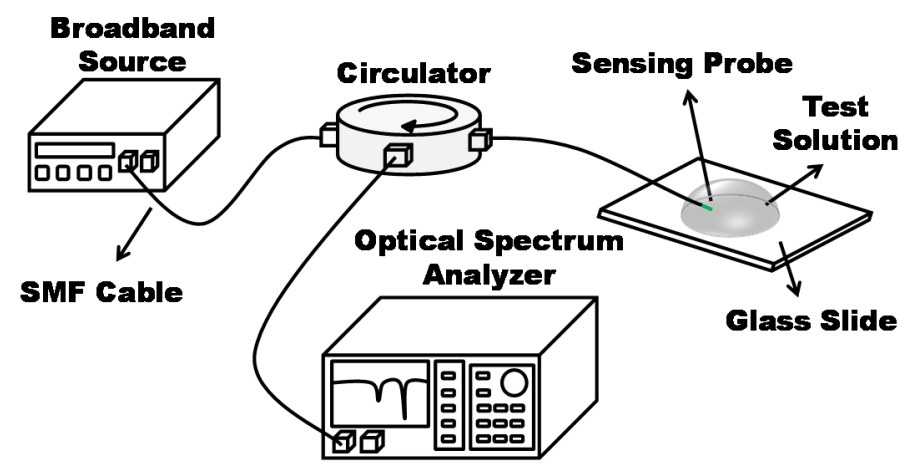

Fig. 3. Experimental setup for the RI measurements with the fabricated sensing probe.

The reflected spectra of the sensor to variations in RI of the ambient environment is shown Fig. 4. It can be seen that the intensity of the reflected spectrum decreased as the RI of the ambient environment increased. The interference dip of the reflected spectrum at $1580 \mathrm{~nm}$ was taken as the reference point for RI sensing measurements

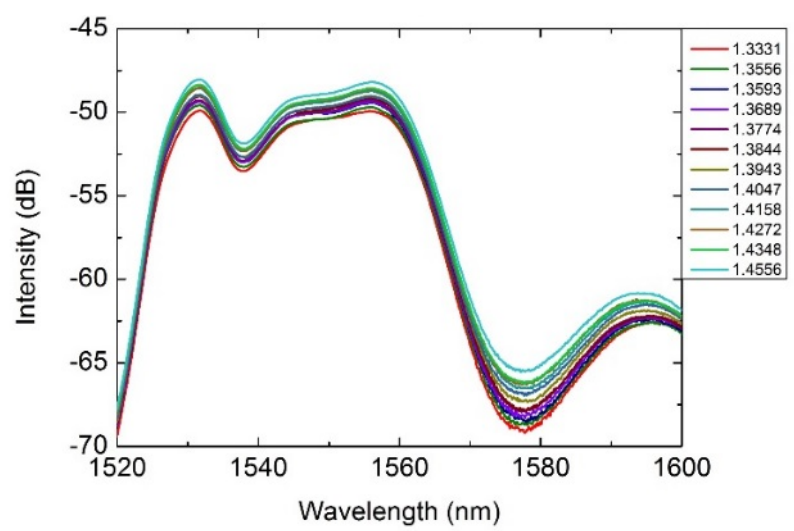

Fig. 4. Output optical spectra of the fibre sensor corresponding to different RI of the ambient environment.

According to the results in Fig. 4, the magnitude of intensity variation is approximately $3.8 \mathrm{~dB}$ within the ambient RI range of 1.33 to 1.46 , and the sensitivity is calculated to be $29.3 \mathrm{~dB} / \mathrm{RIU}$. Considering that the intensity resolution of the OSA is $0.01 \mathrm{~dB}$, the achievable resolution for the sensor can be calculated to be approximately 
$3.4 \times 10^{-4}$ RIU. In order to feed the results into the wireless smart grid platform, the OSA in Fig. 3 is then replaced by an optical tunable bandpass filter followed by an optical amplifier and an optical power meter. The optical bandpass filter is centered at the dip of the spectra shown in Fig. 4. Hence, the change in ambient RI can be registered as a change in the output voltage of the optical power meter instead of the intensity of the output optical spectra so that the WSG platform can process the output data. Fig. 5 plots the output voltage of the optical power meter against the ambient RI of the fibre sensor. The data is then fed into the wireless smart grid platform for transmission to reach to the control center for further processing and analysing.

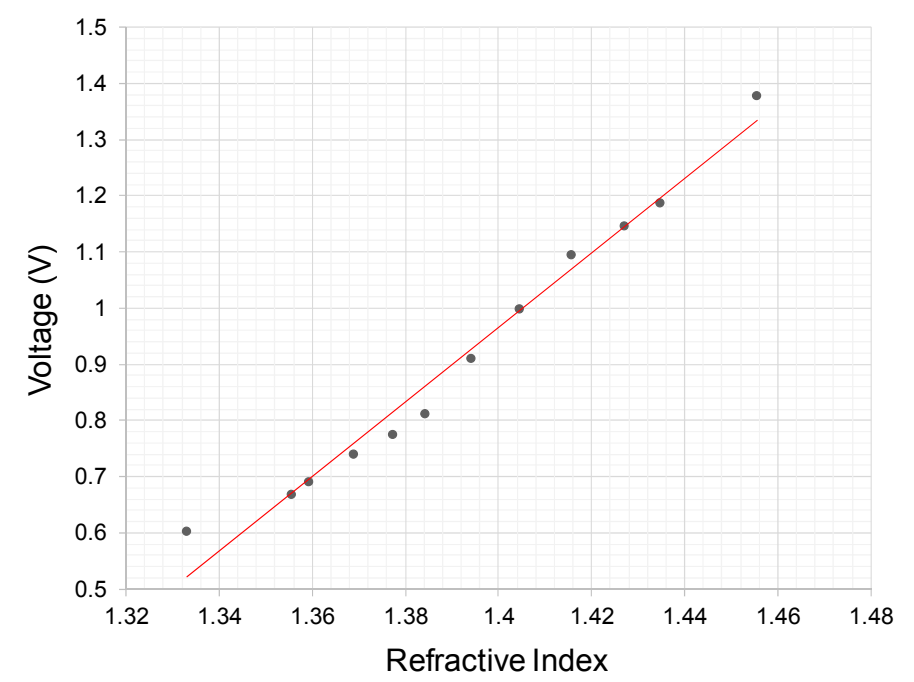

Fig. 5. Plot of the output voltage of the optical power meter corresponding to the various RIs of the external environment.

\section{Conclusion}

In summary, an integration of optical fibre based environmental sensor on wireless smart grid (WSG) platform has been proposed. As a proof-of-concept, a RI sensing probe, fabricated through fusion splicing a multimode fibre (MMF) segment to a single mode fibre (SMF) and depositing carbon nanotubes (CNT) onto the MMF end face of the resulting joint fibre segment, has been presented. The sensing probe displayed clear and distinct variations in its output spectrum to perturbations in RI of its external environment. The achievable sensitivity is calculated to be $29.3 \mathrm{~dB} / \mathrm{RIU}$ for the fabricated fibre sensor within the RI range of 1.33-1.46. The sensing probe also demonstrated good repeatability and stability behaviors as the RI of the external environment varied. The acquired data has been transmitted by the wireless smart meters. Thus, it can allow scalability of the sensing areas. 


\section{References}

1. A. Mahmood, N. Javaid, and S. Razzaq, "A review of wireless communications for smart grid," Renewable and Sustainable Energy Reviews, vol. 41, pp. 248-260, Jan. 2015.

2. Y. Liu, "Wireless Sensor Network Applications in Smart Grid: Recent Trends and Challenges," International Journal of Distributed Sensor Networks, vol. 8, no. 9, p. 492819, Sep. 2012.

3. Z. Guo, F. Ye, J. Guo, Y. Liang, G. Xu, X. Zhang, and Y. Qian, "A wireless sensor network for monitoring smart grid transmission lines," 2014 23rd International Conference on Computer Communication and Networks (ICCCN), Aug. 2014.

4. J. W. Nicholson, R. S. Windeler and D. J. DiGiovanni, "Optically driven deposition of singlewalled carbon-nanotube saturable absorbers on optical fiber end-faces," Opt. Express., vol. 15, no. 15, pp. 9176-9183, Jul. 2007.

5. K. Kashiwagi, S. Yamashita and S. Y. Set, "In-situ monitoring of optical deposition of carbon nanotubes onto fiber end," Opt. Express., vol. 17, no. 7, pp. 5711-5715, Mar. 2009.

6. Y. C. Tan, W. B. Ji, V. Mamidala, K. K. Chow, and S. C. Tjin, "Carbon-nanotube-deposited long period fiber grating for continuous refractive index sensor applications," Sens. Actuators, B, vol. 196, pp. 260-264, Jun. 2014.

7. Y. C. Tan, Z. Q. Tou, V. Mamidala, K. K. Chow, and C. C. Chan, "Continuous refractive index sensing based on carbon-nanotube-deposited photonic crystal fibers," Sens. Actuators, B, vol. 202, pp. 1097-1102, Oct. 2014. 